\title{
Dehydroepiandrosterone reverses chronic hypoxia/reoxygenation-induced right ventricular dysfunction in rats
}

\author{
Eric Dumas de La Roque*,\#,", Nadège Bellance", Rodrigue Rossignol\#, \\ Hugues Begueret*,\#, , Marie Billaud*,*, Pierre Dos Santos ${ }^{\#, \oplus,}$, Thomas Ducret*,*, \\ Roger Marthan*,\#, Diana Dahan*,", David Ramos-Barbón', Óscar Amor-Carro ${ }^{+}$, \\ Jean-Pierre Savineau, ${ }^{\star, \#, \oplus}$ and Michael Fayon,*,*,
}

ABSTRACT: Dehydroepiandrosterone (DHEA) prevents chronic hypoxia-induced pulmonary hypertension and associated right ventricle dysfunction in rats. In this animal model, reoxygenation following hypoxia reverses pulmonary hypertension but not right ventricle dysfunction. We thus studied the effect of DHEA on the right ventricle after reoxygenation, i.e. after a normoxic recovery phase secondary to chronic hypoxia in rats.

Right ventricle function was assessed in vivo by Doppler echocardiography and in vitro by the isolated perfused heart technique in three groups of animals: control, recovery (21 days of hypoxia followed by 21 days of normoxia) and recovery DHEA ( $30 \mathrm{mg} \cdot \mathrm{kg}^{-1}$ every 2 days during the recovery phase). Right ventricle tissue was assessed by optical and electron microscopy.

DHEA abolished right ventricle diastolic dysfunction, as the echographic $E$ wave remained close to that of controls (mean \pm SD $76.5 \pm 2.4$ and $79.7 \pm 1.7 \mathrm{~cm} \cdot \mathrm{s}^{-1}$, respectively), whereas it was diminished to $40.3 \pm 3.7$ in the recovery group. DHEA also abolished right ventricle systolic dysfunction, as shown by the inhibition of the increase in the slope of the pressure-volume curve in isolated heart. The DHEA effect was related to cardiac myocytes proliferation.

In conclusion, DHEA prevents right ventricle dysfunction in this animal model by preventing cardiomyocyte alteration.

KEYWORDS: Cardiac myocyte, chronic hypoxia, dehydroepiandrosterone, mitochondria, pulmonary hypertension

$\mathbf{T}$ ypical chronic lung hypoxaemic diseases, such as chronic obstructive pulmonary disease (COPD) [1], can lead to pulmonary hypertension $(\mathrm{PH})$ and ultimately to right ventricular failure [2-4]. Rodents exposed to chronic hypoxia, either normo- or hyperbaric, consistute a classical animal model used to investigate mechanisms as well as therapeutic targets in this pulmonary vascular disease $[5,6]$. In a rat model of hypobaric chronic hypoxia, we have previously demonstrated that dehydroepiandrosterone (DHEA), an adrenal steroid, prevents and decreases hypoxic $\mathrm{PH}$ and associated right ventricle hypertrophy [7]. In the same animal model, reoxygenation following hypoxia, i.e. a normoxic recovery phase of 21 days of normoxia secondary to a chronic hypoxic period of 21 days, also reverses $\mathrm{PH}$ as it normalises pulmonary pressure and antagonises vascular remodelling [8]. However, such normoxic recovery period does not correct right ventricular dysfunction [9].
We believe that such an experimental model may be relevant in patients suffering from COPD, as they may alternate between successive severe hypoxic episodes related to exacerbations [10] and fewer hypoxic episodes related their oxygen therapy [11].

As mentioned, DHEA has been studied in animal $[7,12]$ and human PH $[13,14]$. More recently, it has been shown that DHEA can modulate cardiac function. DHEA reverses left ventricular stiffness and fibrosis, which typically accompanies ageing in mice [15], and decreases the production of type I collagen by cardiac fibroblasts [16]. DHEA can also modulate oxidative stress in the rat heart [17].

The purpose of this study was thus to evaluate the effect of DHEA on the right ventricle in this chronic hypoxia model followed by reoxygenation, i.e. followed by a normoxic recovery phase in rats. We examined the additional effect of DHEA on reoxygenation of the right ventricular
AFFILIATIONS

*Univ-Bordeaux, Centre de Recherche Cardio-Thoracique U 1045 ,

\#INSERM U 1045, U 688 and U 1034, and

' $\mathrm{CHU}$ de Bordeaux, Bordeaux, France.

+Respiratory Dept, Hospital de la Santa Creu i Sant Pau, Universitat Autònoma de Barcelona, Barcelona, Spain.

CORRESPONDENCE

E. Dumas de la Roque Hôpital Pellegrin Enfants CHU de Bordeaux

Place Amélie Raba Léon 33076 Bordeaux Cedex France

E-mail: edumasdlr@gmail.com

Received:

Jan 212011

Accepted after revision:

March 262012

First published online:

April 202012 
structure and function both in vivo and ex vivo. The signalling pathway activated by DHEA was also determined.

\section{METHODS}

\section{Animal model and DHEA treatment}

The investigation was carried out in agreement with the Guide for the Care and Use of Laboratory Animals [18] and European Directives [19]. Adult male Wistar rats (220-240 g) were randomised into three groups. The first group of rats was kept in a hypobaric chamber for 21 days in order to induce a chronic hypoxic $\mathrm{PH}$ as previously described [7], followed by normoxia for 21 days (recovery group); the second group of rats was also kept in a hypobaric chamber for 21 days followed by normoxia for 21 days while being treated with DHEA (30 $\mathrm{mg} \cdot \mathrm{kg}^{-1}$ every 2 days) during the recovery period (recoveryDHEA group). These two groups were compared with the control group, which was kept at normal atmospheric pressure.

\section{Assessment of heart tissue weight ratio and echocardiography}

The right ventricle hypertrophy index was calculated as the ratio of the weight of the right ventricle to that of the left ventricle plus the septum; the right ventricle mass index was calculated as the ratio of right ventricle weight to bodyweight as previously described [20]. Echocardiography Doppler imaging was performed as previously described [9]. The right ventricle shortening fraction was then estimated as ((enddiastolic diameter - end-systolic diameter $) \times 100$ ) / end-diastolic diameter. The right ventricle diastolic function was estimated by the E-wave and the E/A peak velocity ratio. Pulse-wave Doppler (A and E wave) of the tricuspid valve was recorded in an apical four-chamber view.

\section{Isolated and perfused heart technique}

The isolated and perfused heart technique was performed as previously described [9].

\section{Histological measurements}

Optical and electronmicroscopy were performed as previously described [7]. The right ventricular tissue was fixed in $4 \%(\mathrm{w} / \mathrm{v})$ formaldehyde and $3-\mu \mathrm{m}$ thick sections were stained with haematoxylin, eosin and saffron. The number $(\mathrm{N})$ of myocytes per ventricle was estimated according the following equation: $\mathrm{N}=($ myocyte volume fraction $\times$ ventricular volume $) /$ median myocyte volume [21]. The myocyte volume fraction used was $75 \%$, and ventricular volume was calculated as the ventricular weight divided by the ventricular specific gravity $\left(1.06 \mathrm{~g} \cdot \mathrm{cm}^{-3}\right)$.

\section{Reactive oxygen species measurement}

Reactive oxygen species were measured by means of the electron paramagnetic resonance technique on right ventricle tissue, and was performed as previously described [22].

\section{Mitochondrial activity assays}

Complex 1 and citrate synthase activity assays were performed on right ventricle tissue as previously described [23].

\section{Western blotting}

Western blotting was performed on right ventricle tissue as described previously [23]. Antibodies against respiratory chain complex III core 2 and $\mathrm{F}_{1} \mathrm{~F}_{0}$ adenosine triphosphate (ATP) synthase were obtained from Mitoscience (Eugene, OR, USA). The caspase 3, peroxisome proliferator-activated receptor $\gamma$ coactivator $1 \alpha(\mathrm{PGC} 1 \alpha)$, manganese superoxide dismutase (MnSOD) and endothelial nitric oxide synthase (eNOS) antibodies were obtained from Santa Cruz Biothechnology Inc. (Santa Cruz, CA, USA). The antiphospho-cyclic adenosine monophosphate response element binding (CREB) and antiproliferating cell nuclear antigen (PCNA) were obtained from Abcam Biochemicals (Bristol, UK).

\section{Cell proliferation and apoptosis}

Tissues were fixed in phosphate-buffered $4 \%$ formaldehyde and paraffin embedded. Myocyte cross-sectional areas were measured using an image-based quantitative analysis system (NanoZoomzer Digital Pathology Image software; Hamamatsu Photonics France, Massy, France). The outlines of 50 myocytes were traced in each animal studied. Cell proliferation and apoptosis detection were performed as previously described [24]. Proliferating cardiomyocytes were identified on the basis of PCNA co-immunostaining with desmin. The anti-PCNA monoclonal antibody was obtained from Calbiochem/Merck (Darmstadt, Germany). Apoptotic cardiomyocytes were detected using the ApopTag peroxidase kit (Millipore, Billerica, MA, USA) according to the manufacturer's instructions. Quantitative morphology was blindly performed on coded specimens. The tissue sections were scanned through systematic random field sampling using $0.143-\mathrm{mm}^{2}$ calibrated fields captured with a bright-field microscope and digital image acquisition system (Olympus, Tokyo, Japan). 11-24 (mean \pm SEM 18.1 \pm 1.2 ) fields per specimen were sampled through the complete tissue sections, and the numbers of PCNA+ cell nuclei were referenced to the sampled surface area. Lung tissue sections from a murine asthma model published elsewhere [25] were entered in the staining batches as terminal deoxynucleotidyl transferase deoxyuridine triphosphate nick end labelling (TUNEL)+ control specimens.

\section{Data analysis}

Data values are expressed as the mean \pm SEM. Statistical analyses were performed using the NCSS 5.0 software (NCSS, Kaysville, UT, USA), while intergroup differences were assessed by a Kruskal-Wallis ANOVA, as appropriate. In experiments with comparison of two conditions, as unpaired Mann-Whitney test was used. $\mathrm{n}$ refers to the animal sample size in the relevant experiment. Differences in the data were considered significant when $\mathrm{p}<0.05$.

\section{RESULTS \\ DHEA improves right ventricular dysfunction secondary to the normoxic recovery period}

In vivo, the right ventricular systolic (shortening fraction) and diastolic (tricuspid E and A waves) functions were assessed by Doppler echocardiography ( $n=5$ for each condition). Under basal conditions, there was no difference in the right ventricular shortening fraction between groups. The $\mathrm{E}$ wave was significantly decreased in the recovery group $(40.3 \pm 3.7$ versus $\left.79.7 \pm 1.7 \mathrm{~cm} \cdot \mathrm{s}^{-1}, \mathrm{p}<0.001\right)$ and this effect was significantly prevented by DHEA treatment $\left(76.5 \pm 2.4 \mathrm{~cm} \cdot \mathrm{s}^{-1}\right.$, $\mathrm{p}<0.001$ ) (fig. 1a). Similarly, the E/A wave was significantly increased in the recovery group $(1.3 \pm 0.2$ versus $0.4 \pm 0.01$, $\mathrm{p}<0.001)$ and this effect was significantly prevented by DHEA treatment $(0.4 \pm 0.08, \mathrm{p}<0.001)$ (fig. 1b). 
a)

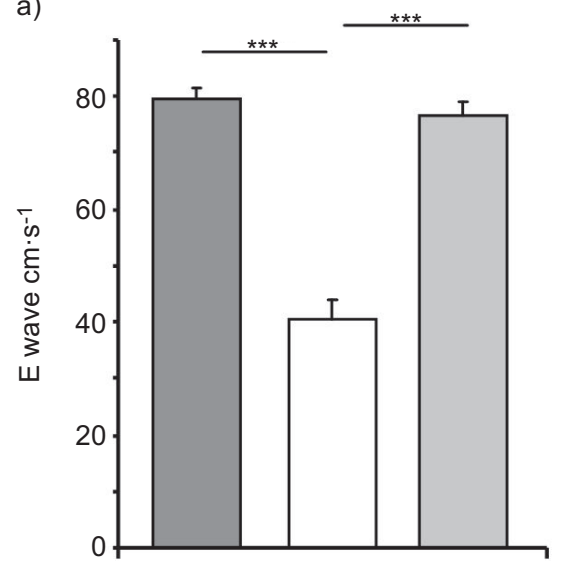

b)

Control

Recovery

Recovery DHEA
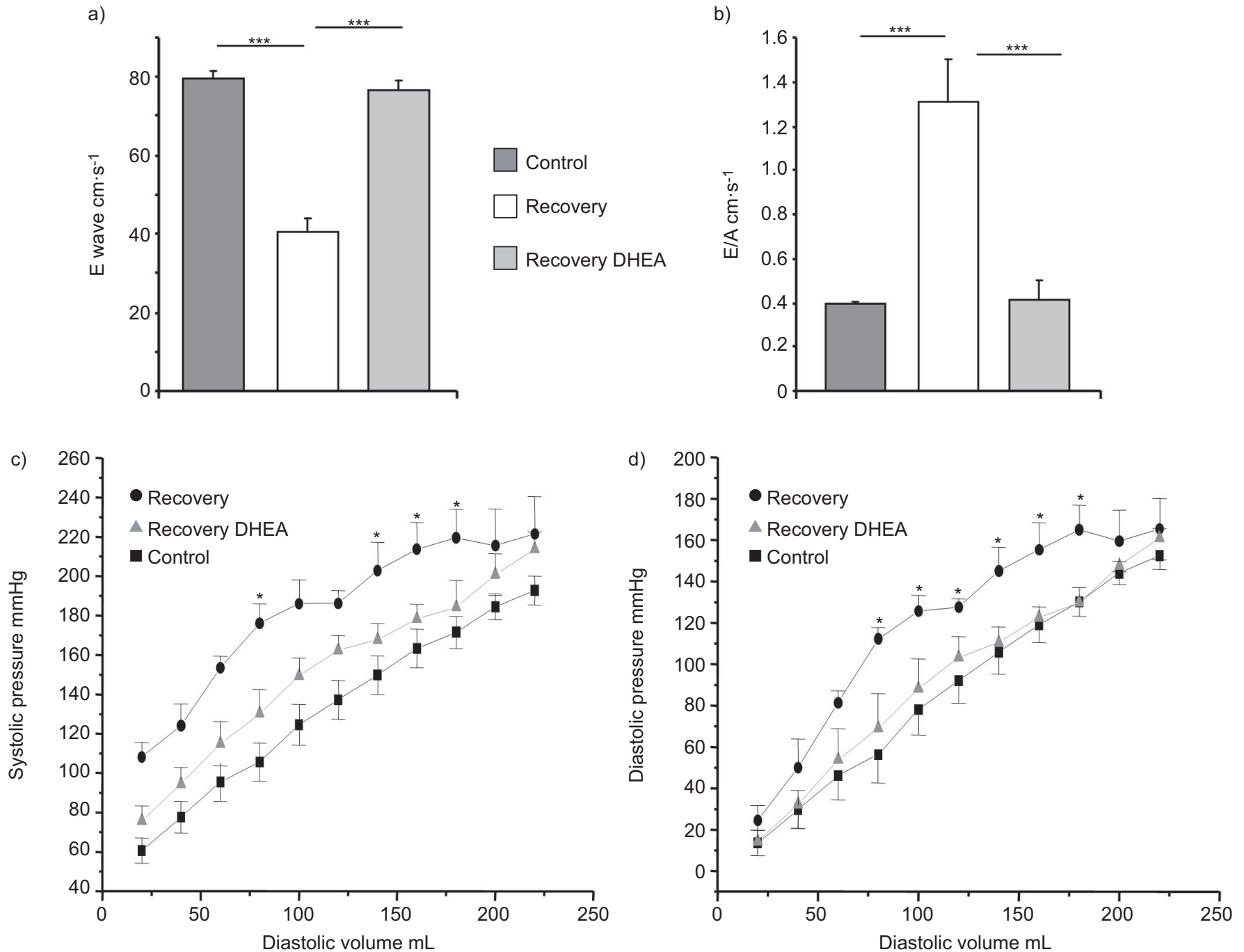

FIGURE 1. Dehydroepiandrosterone (DHEA) improves right ventricle dysfunction observed following a normoxic recovery phase secondary to chronic hypoxia. DHEA (30 $\mathrm{mg} \cdot \mathrm{kg}^{-1}$ every 2 days) was started during the normoxic recovery phase and right ventricle function was evaluated in vivo by Doppler echocardiography and ex vivo by the isolated perfused heart technique ( $\mathrm{n}=5$ per condition). The in vivo right ventricle diastolic function evaluated by a) the $\mathrm{E}$ wave and $\mathrm{b}$ ) the $\mathrm{E} / \mathrm{A}$ ratio was altered in the recovery group; this alteration was prevented by DHEA treatment. The ex vivo evaluation of the right ventricle c) systolic and d) diastolic function showed a positive effect of DHEA on the right ventricle systolic dysfunction (systolic pressure corresponding to the diastolic volumes: 80, 140, 160 and $180 \mathrm{~mL}$ ) and right ventricle diastolic dysfunction (diastolic pressure corresponding to the diastolic volumes: $80-180 \mathrm{~mL}$ ) in the recovery-DHEA group compared with the recovery group. ${ }^{*}: p<0.05 ;{ }^{* \star *}: p<0.001$.

Ex vivo, the right ventricular systolic and diastolic performances were further evaluated in the absence of right ventricular post load using the isolated perfused heart technique ( $\mathrm{n}=5$ for each condition). The recovery condition increased the systolic pressure for large diastolic volumes (80, 140, 160 and $180 \mathrm{~mL} ; \mathrm{p}<0.001$ ) (fig. 1c) and this effect was significantly decreased by DHEA treatment. The recovery condition also increased the diastolic pressure for large diastolic volume $(80-180 \mathrm{~mL}, \mathrm{p}<0.001)$ (fig. 1d) and this effect was also decreased by DHEA.

DHEA improves right ventricle myocyte density during the normoxic recovery period by stimulating cardiomyocyte proliferation

The right ventricle myocyte density (assessed as the number of nuclei per $\mathrm{mm}^{2}$ ) was studied by optical microscopy (nine slides per rat). The mean myocyte density was lower in the recovery group than in the control group $(4.77 \pm 0.33$ versus $8.56 \pm 0.32, \mathrm{p}<0.001)$ and this effect was significantly prevented by DHEA treatment $(6.77 \pm 1.08, \mathrm{p}<0.05)$ (fig. 2$)$. An estimation of the total number of myocytes was also determined and showed the same differences between groups (table 1). In order to determine whether the decrease in myocytes density was related to a loss of myocytes or myocyte hypertrophy, the right ventricle hypertrophy index, the right ventricle mass index and the myocyte cross-sectional area were determined. There was no difference in either index between the recovery and the recovery-DHEA groups (table 1). Moreover there was no difference in the myocyte cross-sectional area between groups (table 1). Therefore, the decrease in myocyte density was related to a loss of myocytes.

To examine the protective effect of DHEA, cell apoptosis was studied by the TUNEL+ staining technique and by Western 

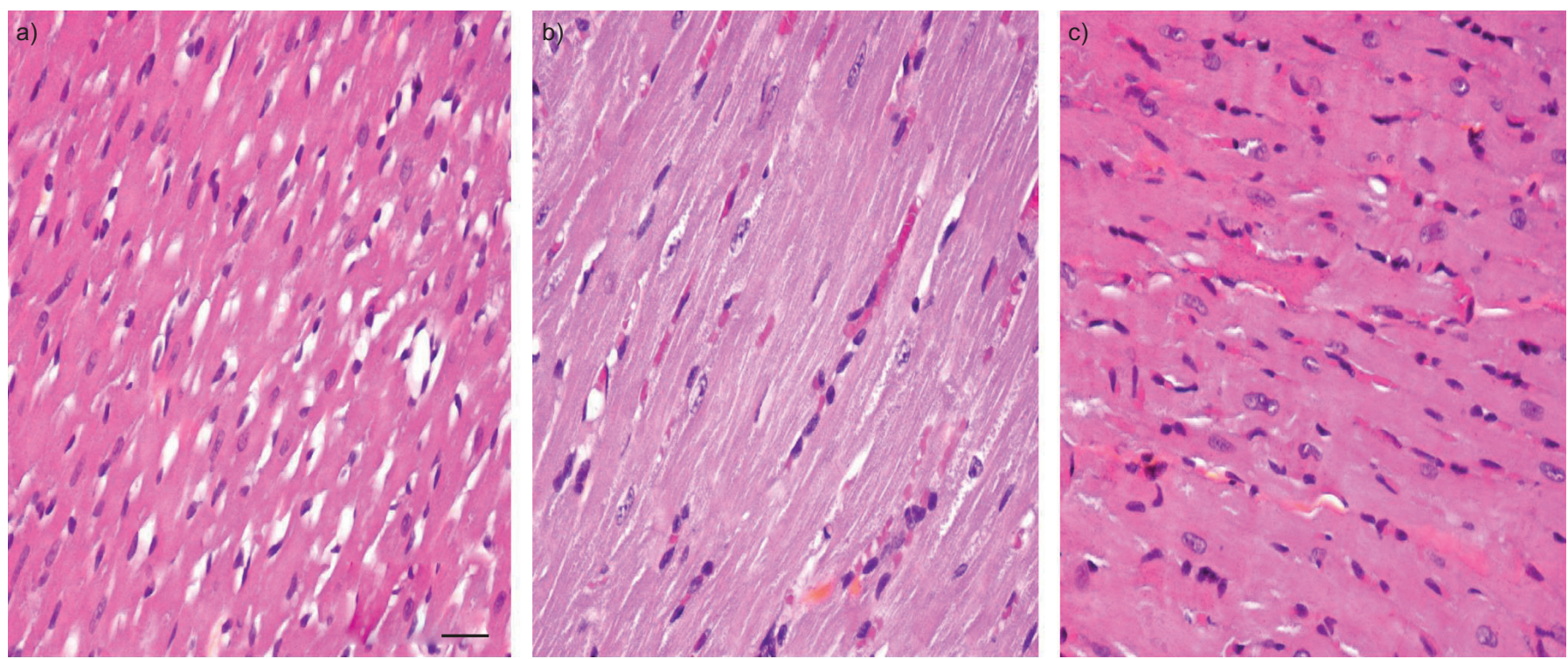

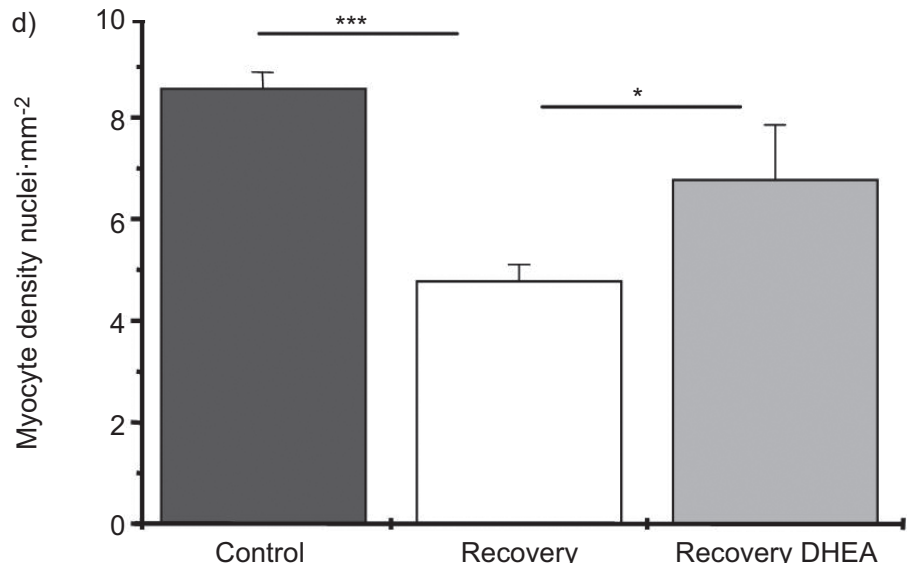

blotting (cleavage of caspase 3). Representative fields of TUNEL+ cells were extremely rare, with no difference between the groups; the Western blot showed no significant difference when comparing caspase 3 activation. The PCNA staining (fig. $3 a-c)$ showed a significant increase of in the recovery-DHEA

\begin{tabular}{|c|c|c|c|}
\hline \multirow[t]{2}{*}{ TABLE 1} & \multicolumn{3}{|c|}{$\begin{array}{l}\text { Effect of dehydroepiandrosterone (DHEA) on } \\
\text { right ventricle myocyte density }\end{array}$} \\
\hline & Control & Recovery & Recovery DHEA \\
\hline Body weight g & $503 \pm 10$ & $470 \pm 18$ & $501 \pm 16$ \\
\hline $\operatorname{CSA} \mu \mathrm{m}^{2}$ & $399 \pm 6$ & $413 \pm 9$ & $416 \pm 6$ \\
\hline $\mathrm{RVMI} \mathbf{m g} \cdot \mathrm{g}^{-1}$ & $0.47 \pm 0.02$ & $0.77 \pm 0.05$ & $0.74 \pm 0.03$ \\
\hline $\mathrm{RVHI} \mathbf{m g} \cdot \mathrm{g}^{-1}$ & $0.23 \pm 0.01$ & $0.36 \pm 0.02$ & $0.35 \pm 0.02$ \\
\hline Myocytes $n \times 10^{6}$ & $42.7 \pm 1.2$ & $35.5 \pm 0.5$ & $41.5 \pm 0.4$ \\
\hline
\end{tabular}

Data are presented as mean \pm SEM. Three animal groups were studied: control, recovery (hypoxia 21 days then normoxia 21 days) and a recovery-DHEA group (30 $\mathrm{mg} \cdot \mathrm{kg}^{-1}$ every 2 days during the recovery phase). CSA: cardiomyocyte cross-sectional area; RVMI: right ventricular mass index; RVHI: right ventricular hypertrophy index.

\begin{abstract}
FIGURE 2. Dehydroepiandrosterone (DHEA) increases right ventricle myocytes density observed following a normoxic recovery phase secondary to chronic hypoxia. DHEA (30 $\mathrm{mg} \cdot \mathrm{kg}^{-1}$ every 2 days) was started during normoxic recovery phase and myocyte density was evaluated by optical histology (nine slides per rat). It showed a decrease in myocyte density secondary to the recovery period, which was significantly improved by DHEA treatment. Typical right ventricle tissue section for each a) control, b) recovery and c) recovery-DHEA group. Scale bar $=50 \mu \mathrm{m}$. d) Summary data for myocyte density expressed as the number of nuclei per $\mathrm{mm}^{2} .{ }^{*}: \mathrm{p}<0.05$ $* * *: p<0.001$
\end{abstract}

group $\left(8.6 \pm 1.6\right.$ cells $\left.\cdot \mathrm{mm}^{-3}\right)$ compared with the recovery group $\left(12.1 \pm 1.8\right.$ cells $\left.\cdot \mathrm{mm}^{-3}, \mathrm{p}<0.05\right)$ and this was confirmed by Western blot (fig. 3d).

\section{DHEA prevents mitochondrial fragmentation induced by normoxic recovery}

In a first set of experiments, the effect of normoxic recovery and DHEA treatment on: 1) the number of mitochondrial sections per cell area; 2) the mitochondrial section area; and 3) the matrix density. Sections were observed at a magnification of $6,000 \times$ in a series of randomly selected tissue sections (nine slides per rat). The number of mitochondrial sections was higher in the recovery group $\left(4.07 \pm 0.20\right.$ versus $2.52 \pm 0.20$ sections $\cdot \mu^{-2}$; $\mathrm{p}<0.001)$ and DHEA significantly reduced this effect $(2.94 \pm$ 0.10 sections $\cdot \mu \mathrm{m}^{-2} ; \mathrm{p}<0.001$ ) (fig. $4 \mathrm{a}-\mathrm{g}$ ). There was no difference in the total mitochondrial section area between the groups. Accordingly, the mean mitochondrial section surface area was lower in the recovery group $(0.099 \pm 0.004$ versus $0.195 \pm$ $\left.0.012 \mu \mathrm{m}^{2} ; \mathrm{p}<0.001\right)$ and DHEA prevented this effect $(0.148 \pm$ $0.003 \mu \mathrm{m}^{2} ; \mathrm{p}<0.05$ ) (fig. 4h). In a second set of experiments, mitochondrial ultrastructural abnormalities including partial cristolysis, disorganised cristae and matrix inclusions were observed at high magnification $(20,500 \times)$. Abnormalities appeared in the recovery group and were less prominent after 

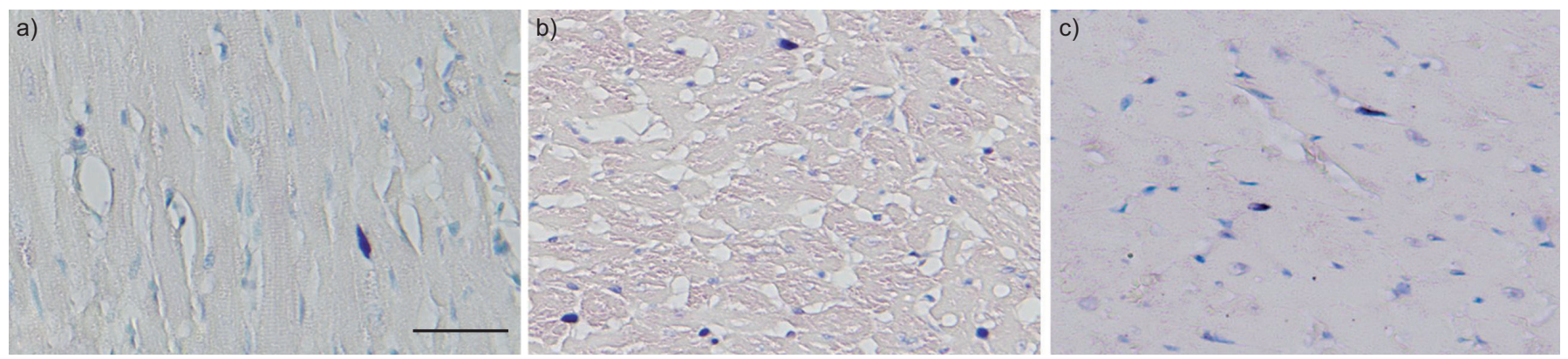

d)
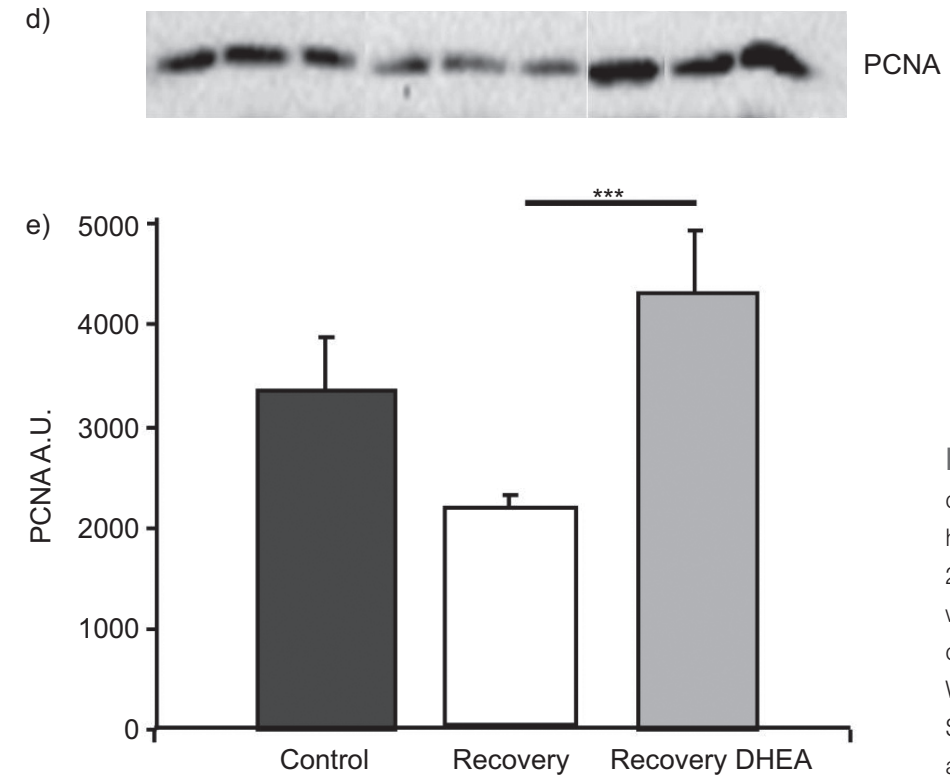

FIGURE 3. Dehydroepiandrosterone (DHEA) increases right ventricle myocyte density observed following a normoxic recovery phase secondary to chronic hypoxia by stimulating cardiomyocyte proliferation. DHEA $\left(30 \mathrm{mg} \cdot \mathrm{kg}^{-1}\right.$ every 2 days) was started during the normoxic recovery phase. Myocyte proliferation was evaluated by proliferating cell nuclear antigen (PCNA) co-immunostaining with desmin staining for a) control, b) recovery and c) recovery-DHEA groups and by d) Western blotting of PCNA, which was significantly improved with DHEA treatment. Scale bar $=50 \mu \mathrm{m}$. d) A typical Western blot (performed in triplicate) for each group and e) summary data. A.U.: arbitrary units. ${ }^{* * *}: p<0.001$

DHEA treatment (fig. 4d-f). The matrix density was also increased following treatment with DHEA as compared with the recovery group (matrix density $216 \pm 85 \%$ in the recovery group, $\mathrm{p}<0.05)$. This suggests a fragmentation of the mitochondrial network during recovery, which was prevented by DHEA.

\section{DHEA protective mechanisms include stimulation of PGC1 $\alpha$ and eNOS via CREB activation}

There was a $35 \pm 7 \%$ increase in PGC $1 \alpha$ with DHEA treatment compared with the level measured after the recovery phase $(\mathrm{p}<0.001)$ (fig. 5a). As it has been recently reported that the expression of PGC1 $\alpha$ can be stimulated in conjunction with the upregulation of eNOS expression in the heart [26], we additionally measured eNOS expression level. As expected, a $53 \pm 17 \%$ increase in the expression level of eNOS was observed in the treated heart $(\mathrm{p}<0.001)$ (fig. $5 b)$. The eNOS gene promoter is controlled by phospho-CREB and is thereby stimulated in adverse situations of increased energy demand [27]. We observed a strong $(136 \pm 18 \%)$ and significant $(\mathrm{p}<0.001)$ increase in the phospho-CREB levels in the DHEAtreated heart samples (fig. 5c).

\section{DHEA improves mitochondrial respiratory chain activity and biogenesis}

We measured the catalytic activity of complex I (reduced nicotinamide adenine dinucleotide-ubiquinone oxidoreductase)

by spectrophotometry. We observed a large increase in complex I activity after the treatment with DHEA ( $p<0.001)$ (fig. 6a). We also measured the activity of citrate synthase that is typically proportional to the respiratory chain protein content [23]. Citrate synthase activity was increased by DHEA treatment to the same extent as complex I activity $(\mathrm{p}<0.001)$ (fig. 6a). The Western blot analysis of respiratory chain complex III and complex $\mathrm{V}\left(\mathrm{F}_{1} \mathrm{~F}_{0}\right.$ ATP synthase) showed an increase in the content of these proteins (by a factor of two, following exposure to DHEA $(p<0.001)$ (fig. 6b). These results suggest that DHEA activates mitochondrial respiratory chain protein expression.

\section{DHEA decreases cellular production of the superoxide anion and stimulates mitochondrial antioxidant defence}

The production of the superoxide anion $\left(\mathrm{O}_{2}{ }^{-}\right)$was slightly increased, although not significantly, in the recovery group in comparison with the control group $(371.9 \pm 48.3 \mathrm{~A} /$ $\left(\mathrm{mg} \cdot \mathrm{mL}^{-1}\right)$ and $261.9 \pm 141.7 \mathrm{~A} /\left(\mathrm{mg} \cdot \mathrm{mL}^{-1}\right)$, respectively). DHEA significantly decreased $\mathrm{O}_{2}^{-}$production $(21.7 \pm 3.7 \mathrm{~A} /$ $\left.\left(\mathrm{mg} \cdot \mathrm{mL}^{-1}\right), \mathrm{p}<0.001\right)$ (fig. 7a). We also measured the expression level of MnSOD that is typically upregulated under pathological conditions of oxidative stress [28]. Accordingly, our results indicate a significant increase in the MnSOD expression level following the recovery procedure with a significant decrease after DHEA treatment $(p<0.001)($ fig. $7 b$ and $\mathrm{c}$ ). 

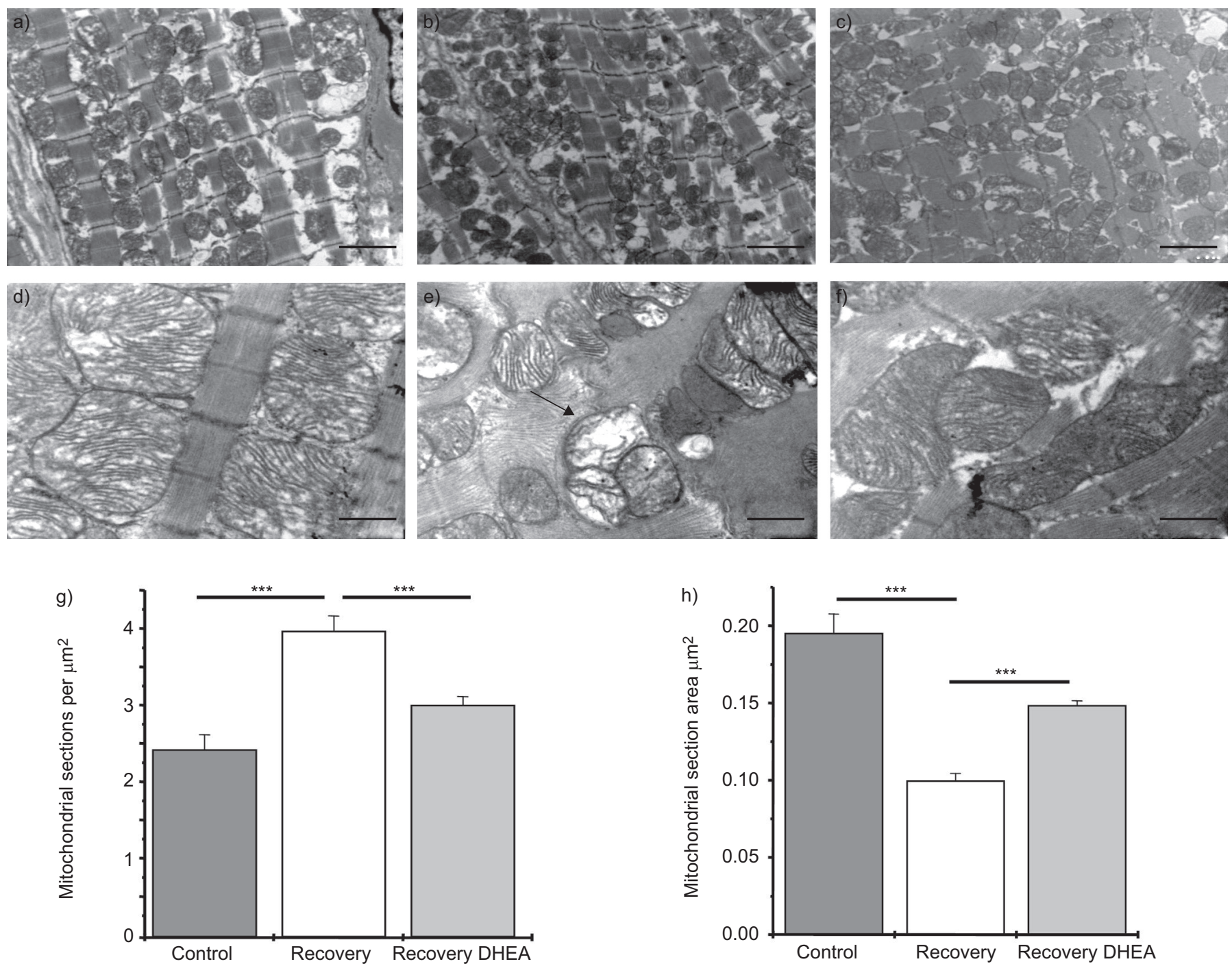

FIGURE 4. Dehydroepiandrosterone (DHEA) prevents mitochondrial fragmentation observed following a normoxic recovery phase secondary to chronic hypoxia. DHEA (30 $\mathrm{mg} \cdot \mathrm{kg}^{-1}$ every 2 days) was started during the normoxic recovery phase and mitochondrial network was evaluated by electron microscopy (a-c; magnification $6,000 \times$, nine slides per rat). It showed a mitochondrial fragmentation (increase in mitochondrial sections $(\mathrm{a}-\mathrm{c}, \mathrm{g})$ and decrease in mitochondrial section area (h)) in the recovery group; this fragmentation was significantly improved by DHEA treatment. Electron micrograph evaluation at larger magnification (d-f; 20,500 $\times$ ) showed mitochondrial ultrastructural abnormalities, including partial cristolysis, disorganised cristae and matrix inclusions in the recovery group; the alterations appeared to be less prominent after treatment with DHEA during the recovery period. Scale bars $=1 \mu \mathrm{m}$. ${ }^{* *}: \mathrm{p}<0.001$

\section{DISCUSSION}

In the present study, we have shown that DHEA improves both systolic and diastolic right ventricle dysfunction observed following a normoxic recovery phase secondary to chronic hypoxia in rats. This right ventricle dysfunction is associated with 1) a significant decrease in cardiac myocyte density and 2) mitochondrial fragmentation without alteration in the respiratory chain activity. DHEA prevented most of these observed modifications. Specifically, DHEA induced both an increase in mitochondrial energy protein expression and a decrease in $\mathrm{O}_{2}{ }^{-}$ production, which was associated with an improvement in mitochondrial antioxidant defence and cardiomyocyte proliferation. DHEA treatment during the recovery had no effect on apoptosis, but stimulated cell proliferation. The signalling pathway of these effects involves an activation of the CREB protein leading to the subsequent expression of eNOS and stimulation of the regulator of mitochondrial biogenesis PGC1 $\alpha$. This suggests a novel mechanism by which DHEA partially antagonises heart alteration following a chronic hypoxia-recovery insult.

To the best of our knowledge, this is the first study exploring the effect of DHEA on right ventricle impairment induced by a normoxic recovery phase following chronic hypoxia exposure. We have previously shown that such a normoxic recovery phase secondary to chronic hypoxia induces nonreversible right ventricular dysfunction and dysplasia in rats [9]. Morphometric analyses demonstrated a mitochondrial network fragmentation that is typically observed in situations of mitochondrial dysfunction [29]. A cardiac dysfunction related to an alteration in mitochondrial structure and activity has been previously described in other models [30,31]. Mitochondrial 
a)
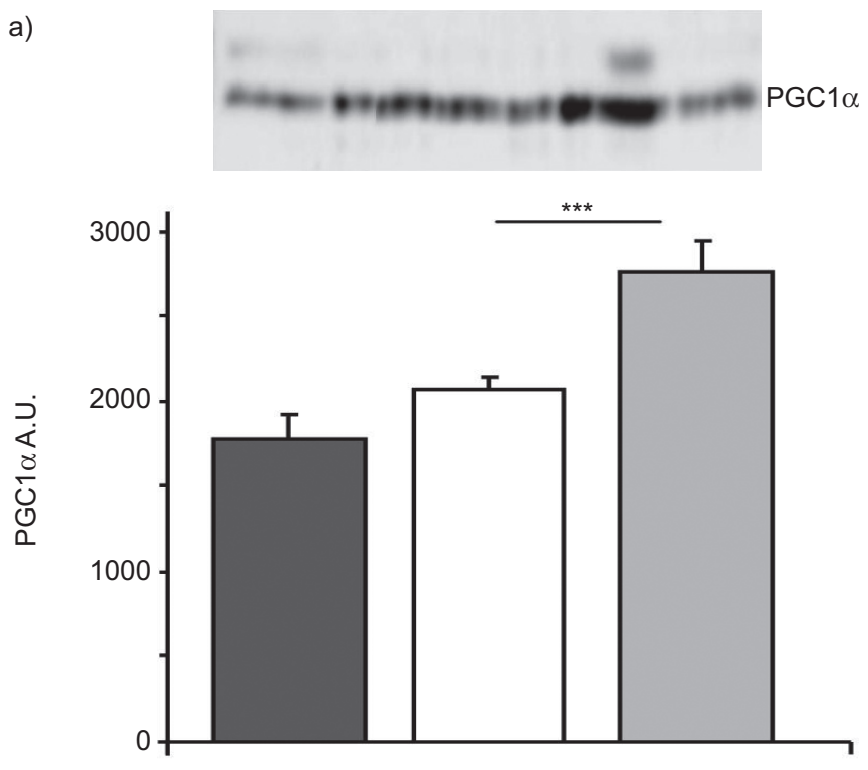

c)
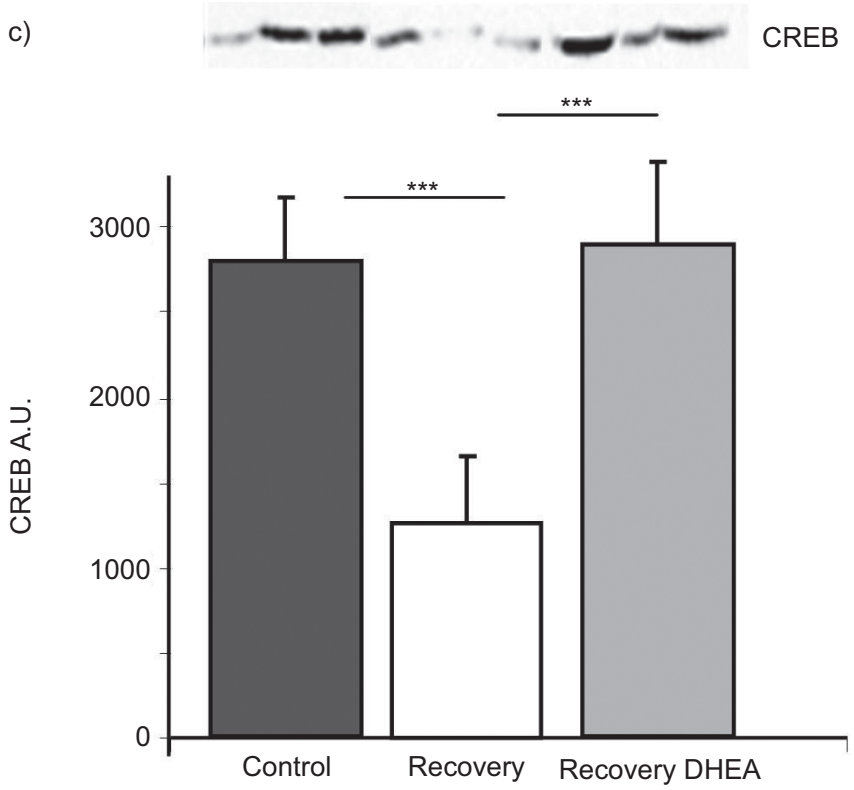

fragmentation could indicate a decrease in cellular ATP levels, as mitochondrial transition from the reticular to the fragmented state is dependent upon ATP levels. The observed decreased cardiac cell density in the right ventricle after the normoxic recovery phase could be explained by the onset of apoptosis during the chronic hypoxia phase [32]. However, such an effect was not observed after the normoxic recovery phase, probably because apoptotic cells are rapidly removed by the immune system.

In the present study, DHEA stimulated mitochondrial respiratory chain protein expression and this effect was associated with an improvement in cell density, which suggests that impairment in mitochondrial energy metabolism did limit cell growth in the recovery group. The alteration in the overall and internal structure of the mitochondria (i.e. fragmentation of the mitochondrial network and cristolysis) observed in the recovery group supports this hypothesis. In the DHEA-treated b)
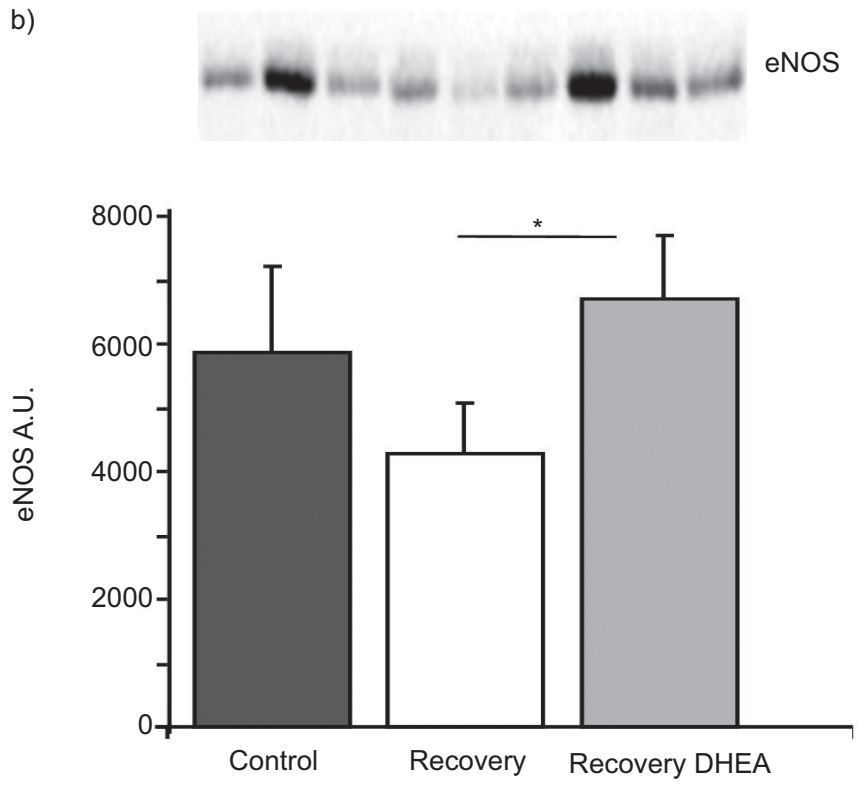

FIGURE 5. Dehydroepiandrosterone (DHEA) protective mechanisms include the stimulation of peroxisome proliferator-activated receptor $\gamma$ coactivator $1 \alpha$ (PGC1 $\alpha$ ), endothelial nitric oxide synthase (eNOS) and cyclic AMP response element binding (CREB). DHEA (30 $\mathrm{mg} \cdot \mathrm{kg}^{-1}$ every 2 days) was started during the normoxic recovery phase secondary to chronic hypoxia. Western blot evaluation of a) PGC1 $\alpha$ showed an increase in expression after DHEA treatment $(p<0.001)$, b) eNOS showed a decrease in expression in the recovery group that was prevented by DHEA treatment and c) phospho-CREB showed a decrease in expression in the recovery group that was prevented by DHEA treatment. Typical Western blots are shown in triplicate for each group a-c). Graphs show summary data. A.U.: arbitrary units. ${ }^{*}: p<0.05 ;{ }^{* * *}: p<0.001 . p<0.001$.

group, mitochondrial configuration returned to normal and oxidative phosphorylation proteins were upregulated. Taken together, these observations suggest that DHEA stimulates mitochondrial biogenesis in the right ventricle, as has previously been shown in the liver and the brain [33, 34].

Our study also highlights the cellular effect of DHEA and the associated signalling pathways in the right ventricle. PGC1 $\alpha$ is a transcriptional co-activator implicated in mitochondrial biogenesis $[35,36]$ that can be stimulated in conjunction with the upregulation of eNOS expression in the heart [26]. The mechanism of such upregulation of eNOS may be due to the DHEA-induced increase in CREB, a factor controlling eNOS gene promoter. Taken together, these data indicate a possible implication of the eNOS/PGC1 $\alpha /$ CREB pathway in the effects of DHEA. Interestingly, a recent study [37] indicates that PGC1 $\alpha$ controls DHEA synthesis in the liver. Together with our observations, this could indicate that DHEA participates in 


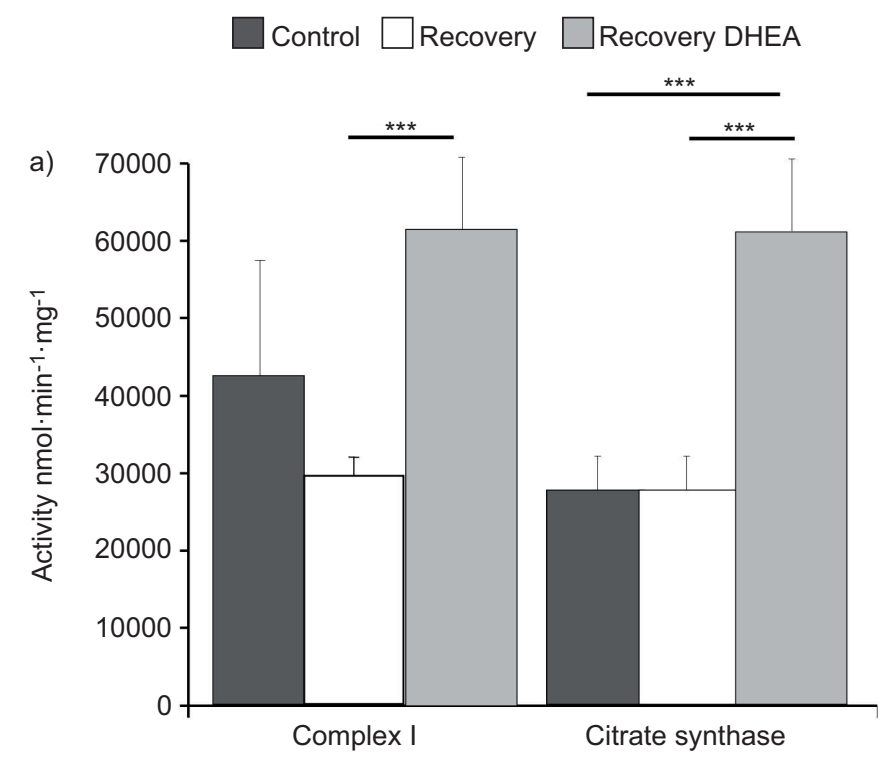

b)
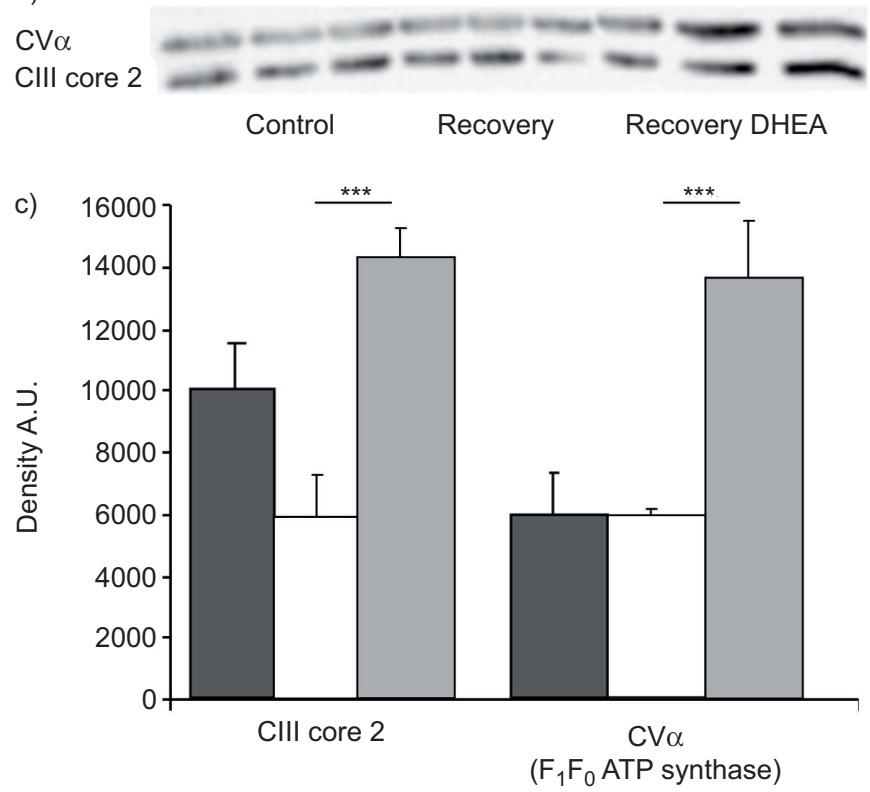

FIGURE 6. Dehydroepiandrosterone (DHEA) improves mitochondrial respiratory chain activity and organellar biogenesis. DHEA (30 mg $\cdot \mathrm{kg}^{-1}$ every 2 days) was started during the normoxic recovery phase secondary to chronic hypoxia. The mitochondrial respiratory chain activity was evaluated by spectrophotometry and the biogenesis by Western blotting. a) The catalytic activity of complex I (reduced nicotinamide adenine dinucleotide-ubiquinone oxidoreductase) and citrate synthase, measured by spectrophotometry), were largely increased after treatment with DHEA. b, c) Western blot evaluation of respiratory chain complex III and complex $V\left(F_{1} F_{0}-A T P s y n t h a s e\right)$ showed a large increase in protein expression after DHEA treatment. b) A typical Western blot in triplicate for each group; c) summary data. A.U.: arbitrary units; CIII: complex III; CV: complex V. ${ }^{* *}$ : $p<0.001$.

feedback control of PGC1 $\alpha$ expression or activation. A feedback control of PGC1 $\alpha$ expression by its target genes would be a remarkable feature of the transcriptional network and regulatory signals that govern mitochondrial biogenesis. Furthermore, we show that DHEA decreases $\mathrm{O}_{2}^{-}$production. This could be one of the mechanisms underlying the protective

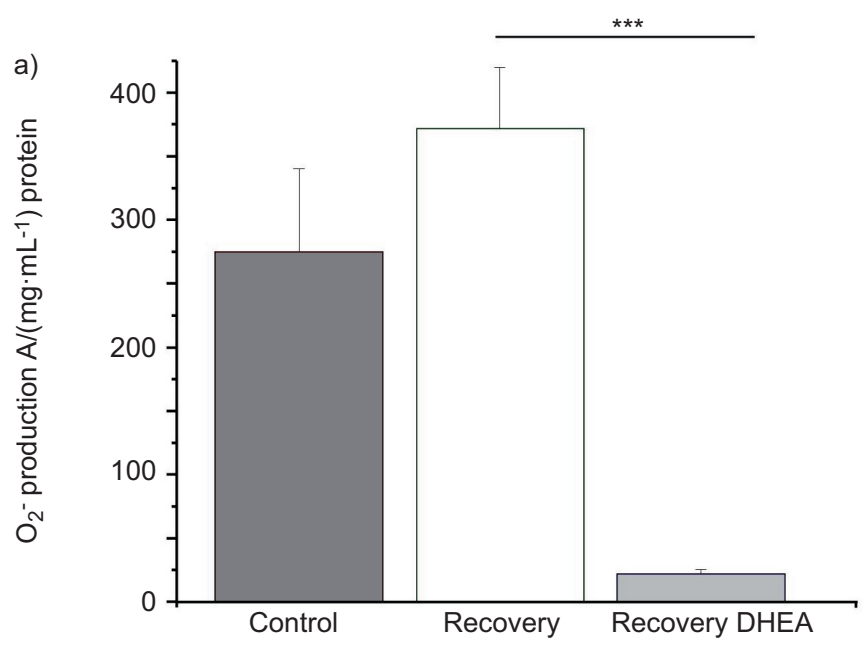

b)

MnSOD
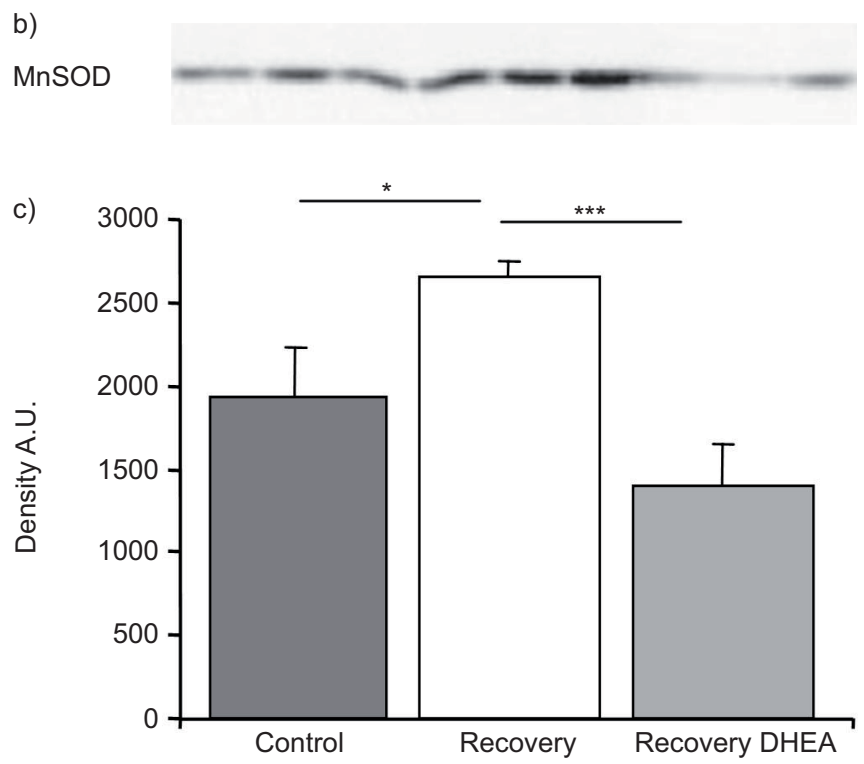

FIGURE 7. Dehydroepiandrosterone (DHEA) decreases cellular production of the superoxide anion $\left(\mathrm{O}_{2}{ }^{-}\right)$and stimulates mitochondrial antioxidant defences. DHEA (30 mg $\cdot \mathrm{kg}^{-1}$ every 2 days) was started during the normoxic recovery phase secondary to chronic hypoxia. Production of superoxide anion was evaluated by the electron paramagnetic resonance (EPR) method. DHEA treatment induced a decrease in superoxide anion production. a) Data from EPR spectrometry are expressed as a ratio of the amplitude of the pic (A) out of the protein concentration of each pool of tissue in $\mathrm{mg} \cdot \mathrm{mL}^{-1}$. b, c) Mitochondrial antioxidant defences was assessed by b) Western blot evaluation of manganese superoxide dismutase (MnSOD) expression. It showed an increase in protein expression in the recovery group, which was significantly decreased after DHEA treatment. b) A typical Western blot (performed in triplicate) for each group; c) shows summary data. A.U. arbitrary units. ${ }^{*}: p<0.05 ; * *: p<0.001$

effect of DHEA. Indeed, reactive oxygen species are known to be involved in mitochondrial damage and cellular injury, and may lead to heart failure $[2,38]$. Moreover, the MnSOD level was higher during the normoxic recovery phase (where oxidative stress was measured) and decreased with DHEA treatment. Our data therefore support previous observations that $\mathrm{MnSOD}$ expression is controlled by the reactive oxygen species concentration [39]. 
In conclusion, DHEA prevents right ventricle dysfunction, remodelling and myocyte alteration observed following a normoxic recovery phase secondary to chronic hypoxia. The stimulation of the antioxidant defence and of the mitochondrial metabolism via an activation of the eNOS/PGC1 $\alpha / C R E B$ axis may explain the decrease in reactive oxygen species production and the increase in cell proliferation observed after DHEA treatment. 1) Such an experimental model may be relevant in patients suffering from COPD and $\mathrm{PH}$ because they alternate between successive severe hypoxic episodes related to exacerbations and less hypoxic episodes related their oxygen therapy, and 2) DHEA is well tolerated [40] and is under current investigation in PH in humans [14]. Thus, DHEA may prove to be a valuable molecule that deserves further studies, particularly in $\mathrm{PH}$ secondary to COPD, where right ventricular failure is one of the main causes of mortality.

\section{STATEMENT OF INTEREST}

None declared.

\section{ACKNOWLEDGEMENTS}

The authors want to thank A-M. Lomenech, P. Techoueyres, S. Vautrat, G. Simon, S. Guerit and L. Parios (all Inserm U1045, Bordeaux, France) for their technical assistance.

\section{REFERENCES}

1 Marthan R, Castaing Y, Manier G, et al. Gas exchange alterations in patients with chronic obstructive lung disease. Chest 1985; 87: 470-475.

2 Bogaard HJ, Abe K, Vonk Noordegraaf A, et al. The right ventricle under pressure: cellular and molecular mechanisms of right-heart failure in pulmonary hypertension. Chest 2009; 135: 794-804.

3 Chaouat A, Naeije R, Weitzenblum E. Pulmonary hypertension in COPD. Eur Respir J 2008; 32: 1371-1385.

4 Weitzenblum E, Chaouat A, Canuet M, et al. Pulmonary hypertension in chronic obstructive pulmonary disease and interstitial lung diseases. Semin Respir Crit Care Med 2009; 30: 458-470.

5 Naeije R, Dewachter L. Animal models of pulmonary arterial hypertension. Rev Mal Respir 2007; 24: 481-496.

6 Stenmark KR, Meyrick B, Galie N, et al. Animal models of pulmonary arterial hypertension: the hope for etiological discovery and pharmacological cure. Am J Physiol Lung Cell Mol Physiol 2009; 297: L1013-L1032.

7 Bonnet S, Dumas-de-La-Roque E, Begueret $\mathrm{H}$, et al. Dehydroepiandrosterone (DHEA) prevents and reverses chronic hypoxic pulmonary hypertension. Proc Natl Acad Sci USA 2003; 100: 9488-9493.

8 Bonnet S, Dubuis E, Vandier C, et al. Reversal of chronic hypoxiainduced alterations in pulmonary artery smooth muscle electromechanical coupling upon air breathing. Cardiovasc Res 2002; 53: 1019-1028

9 Bonnet P, Bonnet S, Boissiere J, et al. Chronic hypoxia induces nonreversible right ventricle dysfunction and dysplasia in rats. Am J Physiol Heart Circ Physiol 2004; 287: H1023-H1028.

10 Barbera JA, Roca J, Ferrer A, et al. Mechanisms of worsening gas exchange during acute exacerbations of chronic obstructive pulmonary disease. Eur Respir J 1997; 10: 1285-1291.

11 Moore RP, Berlowitz DJ, Denehy L, et al. A randomised trial of domiciliary, ambulatory oxygen in patients with COPD and dyspnoea but without resting hypoxaemia. Thorax 2011; 66: 32-37.

12 Hampl V, Bibova J, Povysilova V, et al. Dehydroepiandrosterone sulphate reduces chronic hypoxic pulmonary hypertension in rats. Eur Respir J 2003; 21: 862-865.

13 Dumas de la Roque E, Savineau JP, Bonnet S. Dehydroepiandrosterone: a new treatment for vascular remodeling diseases including pulmonary arterial hypertension. Pharmacol Ther 2010; 126: 186-199.

14 Dumas de La Roque E, Savineau JP, Metivier AC, et al. Dehydroepiandrosterone (DHEA) improves pulmonary hypertension in chronic obstructive pulmonary disease (COPD): a pilot study. Ann Endocrinol (Paris) 2012; 73: 20-25.

15 Alwardt CM, Yu Q, Brooks HL, et al. Comparative effects of dehydroepiandrosterone sulfate on ventricular diastolic function with young and aged female mice. Am J Physiol Regul Integr Comp Physiol 2006; 290: R251-R256.

16 Iwasaki T, Mukasa K, Yoneda M, et al. Marked attenuation of production of collagen type I from cardiac fibroblasts by dehydroepiandrosterone. Am J Physiol Endocrinol Metab 2005; 288: E1222-E1228.

17 Jacob MH, Janner Dda R, Bello-Klein A, et al. Dehydroepiandrosterone modulates antioxidant enzymes and Akt signaling in healthy Wistar rat hearts. J Steroid Biochem Mol Biol 2008; 112: 138-144.

18 National Institutes of Health. Guide for the Care and Use of Laboratory Animals, 85-23. Bethesda, NIH Publications, 1996.

19 European Union. Council directive 86/609/EEC of 24 November 1986 on the approximation of laws, regulations, and administrative provisions of the Member States regarding the protection of animals used for experimental and other scientific purposes. Official J Eur Union 1986; L358: 1-28.

20 Yang DL, Zhang HG, Xu YL, et al. Resveratrol inhibits right ventricular hypertrophy induced by monocrotaline in rats. Clin Exp Pharmacol Physiol 2010; 37: 150-155.

21 Colomer JM, Terasawa M, Means AR. Targeted expression of calmodulin increases ventricular cardiomyocyte proliferation and deoxyribonucleic acid synthesis during mouse development. Endocrinology 2004; 145: 1356-1366.

22 Billaud M, Marthan R, Savineau JP, et al. Vascular smooth muscle modulates endothelial control of vasoreactivity via reactive oxygen species production through myoendothelial communications. PLoS One 2009; 4: e6432.

23 Benard G, Faustin B, Passerieux E, et al. Physiological diversity of mitochondrial oxidative phosphorylation. Am J Physiol Cell Physiol 2006; 291: C1172-C1182.

24 Ramos-Barbon D, Presley JF, Hamid QA, et al. Antigen-specific CD4+ $\mathrm{T}$ cells drive airway smooth muscle remodeling in experimental asthma. J Clin Invest 2005; 115: 1580-1589.

25 Fraga-Iriso R, Nunez-Naveira L, Brienza NS, et al. Development of a murine model of airway inflammation and remodeling in experimental asthma. Arch Bronconeumol 2009; 45: 422-428.

26 Nisoli E, Tonello C, Cardile A, et al. Calorie restriction promotes mitochondrial biogenesis by inducing the expression of eNOS. Science 2005; 310: 314-317.

27 Vankoningsloo S, De Pauw A, Houbion A, et al. CREB activation induced by mitochondrial dysfunction triggers triglyceride accumulation in 3T3-L1 preadipocytes. J Cell Sci 2006; 119: 1266-1282.

28 Wallace DC. Animal models for mitochondrial disease. Methods Mol Biol 2002; 197: 3-54.

29 Benard G, Rossignol R. Ultrastructure of the mitochondrion and its bearing on function and bioenergetics. Antioxid Redox Signal 2008; 10: 1313-1342.

30 Makino A, Suarez J, Gawlowski T, et al. Regulation of mitochondrial morphology and function by O-GlcNAcylation in neonatal cardiac myocytes. Am J Physiol Regul Integr Comp Physiol 2011; 300: R1296-1302.

31 Camara AK, Bienengraeber M, Stowe DF. Mitochondrial approaches to protect against cardiac ischemia and reperfusion injury. Front Physiol 2011; 2: 13.

32 Bhuiyan MS, Fukunaga K. Inhibition of HtrA2/Omi ameliorates heart dysfunction following ischemia/reperfusion injury in rat heart in vivo. Eur J Pharmacol 2007; 557: 168-177. 
33 Patel MA, Katyare SS. Treatment with dehydroepiandrosterone (DHEA) stimulates oxidative energy metabolism in the liver mitochondria from developing rats. Mol Cell Biochem 2006; 293: 193-201.

34 Patel MA, Katyare SS. Dehydroepiandrosterone (DHEA) treatment stimulates oxidative energy metabolism in the cerebral mitochondria from developing rats. Int J Dev Neurosci 2006; 24: 327-334.

35 Lehman JJ, Barger PM, Kovacs A, et al. Peroxisome proliferatoractivated receptor gamma coactivator-1 promotes cardiac mitochondrial biogenesis. J Clin Invest 2000; 106: 847-856.

36 Gerhart-Hines Z, Rodgers JT, Bare O, et al. Metabolic control of muscle mitochondrial function and fatty acid oxidation through SIRT1/PGC-1 $\alpha$. EMBO J 2007; 26: 1913-1923.
37 Grasfeder LL, Gaillard S, Hammes SR, et al. Fasting-induced hepatic production of DHEA is regulated by PGC- $1 \alpha$, ERR $\alpha$, and HNF4 $\alpha$. Mol Endocrinol 2009; 23: 1171-1182.

38 Tsutsui H, Kinugawa S, Matsushima S. Mitochondrial oxidative stress and dysfunction in myocardial remodelling. Cardiovasc Res 2009; 81: 449-456.

39 Rohrdanz E, Schmuck G, Ohler S, et al. Changes in antioxidant enzyme expression in response to hydrogen peroxide in rat astroglial cells. Arch Toxicol 2001; 75: 150-158.

40 Dyner TS, Lang W, Geaga J, et al. An open-label dose-escalation trial of oral dehydroepiandrosterone tolerance and pharmacokinetics in patients with HIV disease. J Acquir Immune Defic Syndr 1993; 6: 459-465. 